\title{
ChemComm
}

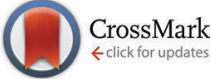

Cite this: Chem. Commun., 2017. 53,1064

Received 18th October 2016, Accepted 17th December 2016

DOI: $10.1039 / c 6 c c 08372 b$

www.rsc.org/chemcomm

\section{Development of a reversible fluorescent probe for reactive sulfur species, sulfane sulfur, and its biological application $\dagger$}

\author{
Yoko Takano, ${ }^{a}$ Kenjiro Hanaoka, ${ }^{* a}$ Kazuhito Shimamoto, ${ }^{a}$ Ryo Miyamoto, ${ }^{b}$ \\ Toru Komatsu, ${ }^{\text {ac }}$ Tasuku Ueno, ${ }^{a}$ Takuya Terai, ${ }^{a}$ Hideo Kimura, ${ }^{b}$ Tetsuo Nagano ${ }^{d}$ \\ and Yasuteru Urano*aef
}

\begin{abstract}
We report a reversible off/on fluorescent probe for monitoring concentration changes of sulfane sulfur by utilizing the unique ability of sulfane sulfur to bind reversibly to other sulfur atoms and the intramolecular spirocyclization reaction of xanthene dyes. It reversibly visualized sulfane sulfur in living A549 cells and primary-cultured hippocampal astrocytes.
\end{abstract}

Sulfane sulfur is one of reactive sulfur species, and is a form of sulfur with six valence electrons and no charge $\left(S^{0}\right)$. Various sulfane sulfur molecules exist in biological systems, in the forms of persulfides (R-S-SH), polysulfides ( $\left.\mathrm{R}-\mathrm{S}-\mathrm{S}_{n}-\mathrm{S}-\mathrm{R}\right)$, hydrogen polysulfides $\left(\mathrm{H}_{2} \mathrm{~S}_{n}\right)$, and protein-bound cysteine persulfide. ${ }^{1}$ Low-molecular-weight persulfides such as cysteine and glutathione persulfides exist at $\mu \mathrm{M}$ order concentration inside cells. ${ }^{2}$ Persulfides are highly reducing and nucleophilic, and therefore they are capable of scavenging oxidants and intracellular electrophiles. Further, some physiological phenomena that were originally attributed to the bioactivity of $\mathrm{H}_{2} \mathrm{~S}$ may actually be mediated by sulfane sulfur, though this remains controversial; ${ }^{3}$ one example is the S-sulfhydration posttranslational modification, which was considered to be due to $\mathrm{H}_{2} \mathrm{~S}$ activity. ${ }^{4}$ However, recent studies have demonstrated that persulfides are more effective than $\mathrm{H}_{2} \mathrm{~S}$

\footnotetext{
${ }^{a}$ Graduate School of Pharmaceutical Sciences, The University of Tokyo, 7-3-1, Hongo, Bunkyo-ku, Tokyo 113-o033, Japan. E-mail: uranokun@m.u-tokyo.ac.jp, khanaoka@mol.f.u-tokyo.ac.jp

${ }^{b}$ Department of Molecular Pharmacology, National Institute of Neuroscience, National Center of Neurology and Psychiatry, 4-1-1 Ogawa-Higashi, Kodaira, Tokyo 187-8502, Japan

${ }^{c}$ PRESTO, Japan Science and Technology Agency (JST), 4-1-8 Honcho, Kawaguchi, Saitama 332-0012, Japan

${ }^{d}$ Drug Discovery Initiative, The University of Tokyo, 7-3-1, Hongo, Bunkyo-ku, Tokyo 113-0033, Japan

${ }^{e}$ Graduate School of Medicine, The University of Tokyo, 7-3-1 Hongo, Bunkyo-ku, Tokyo 113-0033, Japan

${ }^{f}$ AMED CREST (Japan) Agency for Medical Research and Development 1-7-1 Otemachi, Chiyoda-ku, Tokyo 100-0004, Japan

$\dagger$ Electronic supplementary information (ESI) available: Synthesis, experimental details, characterization of compounds, in vitro absorption and fluorescence spectra measurements and the fluorescence imaging of live cells. See DOI: $10.1039 / \mathrm{c} 6 \mathrm{cc} 08372 \mathrm{~b}$
}

for S-sulfhydration; for example, Dick et al. reported that lipid phosphatase PTEN (phosphatase and tensin homolog) was oxidized by polysulfide, resulting in its inhibition. ${ }^{5}$ It is also reported that sulfane sulfur species are biosynthesized by three enzymes which have generally been regarded as $\mathrm{H}_{2} \mathrm{~S}$-producers. Akaike et al. demonstrated that cystathionine $\gamma$-lyase (CSE) and cystathionine $\beta$-synthase (CBS) are capable of directly generating cysteine persulfide, Cys-SSH, from cystine, ${ }^{2}$ and Kimura et al. reported that $\mathrm{H}_{2} \mathrm{~S}_{3}$ and $\mathrm{H}_{2} \mathrm{~S}_{2}$, as well as $\mathrm{H}_{2} \mathrm{~S}$, are produced from 3-mercaptopyruvate (3MP) by 3-mercaptopyruvate sulfurtransferase (3MST) in the brain. ${ }^{6}$

The increasing recognition of the importance of sulfane sulfur in biological systems has led to the development of various detection methods for sulfane sulfur. The unique chemical characteristics of sulfane sulfur species have been utilized in fluorescent probe design. For example, persulfides are more acidic and nucleophilic than the corresponding thiols by $1-2 \mathrm{p} K_{\mathrm{a}}$ units due to the $\alpha$-effect. ${ }^{7,8}$ On the other hand, tautomerization of persulfide to thiosulfoxide also generates reactive species that are very electrophilic and highly reactive to nucleophiles. Moreover, sulfane sulfur can reversibly bind to other sulfur atoms. Based on these characteristics, fluorescent probes such as the SSP, DSP and PSP series have recently been developed by Xian et al. to detect sulfane sulfur selectively in terms of a fluorescence increase. ${ }^{9-11}$ However, these probes react irreversibly, and therefore they cannot visualize the dynamics of intracellular sulfane sulfur. So, we set out to develop a reversible fluorescent probe to monitor the intracellular dynamics of sulfane sulfur.

For this purpose, we focused on two facts: (1) the unique ability of sulfane sulfur to reversibly bind to other sulfur atoms and (2) the intramolecular spirocyclization reaction of xanthene dyes. As shown in Fig. 1a, we anticipated that the introduction of a thiol group into the benzene moiety of xanthene dyes would allow persulfide to be generated by transfer of sulfur from sulfane sulfur species to the thiol group, and then intramolecular spirocyclization would occur because of the high nucleophilicity of persulfide. Indeed, we found that 2-thio RB showed an absorbance decrease in the visible region upon addition of $50 \mu \mathrm{M}$ 

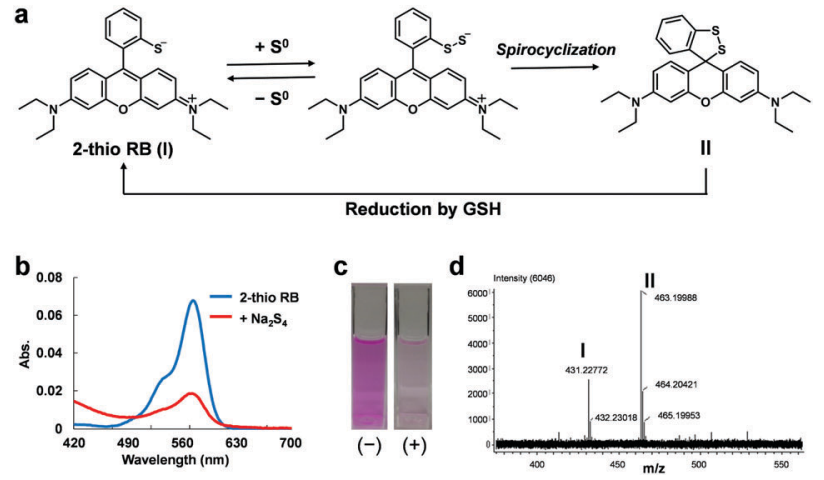

Fig. 1 (a) Proposed mechanism of the intramolecular spirocyclization and the ring-opening reaction of 2-thio RB. (b) Absorption spectra of $1 \mu \mathrm{M}$ 2-thio RB before (blue) and after (red) reaction with $50 \mu \mathrm{M} \mathrm{Na}_{2} \mathrm{~S}_{4}$. Spectra were measured in $100 \mathrm{mM}$ sodium phosphate buffer $(\mathrm{pH} 7.4)$ containing $100 \mu \mathrm{M} \mathrm{GSH}$ to reduce the intermolecular disulfide bond of dimerized 2-thio RB. Cosolvent: 0.1\% DMSO. (c) Photos of 2-thio RB before (-) and after (+) reaction with $50 \mu \mathrm{M} \mathrm{Na}_{2} \mathrm{~S}_{4}$. (d) ESI-MS spectrum of the reaction mixture of 2-thio RB and $\mathrm{Na}_{2} \mathrm{~S}_{4}$. Peaks I and II were assigned to 2-thio RB $\left([\mathrm{M}]^{+}\right)$and its spirocyclized form $\left([\mathrm{M}+\mathrm{H}]^{+}\right)$shown in (a), respectively.

$\mathrm{Na}_{2} \mathrm{~S}_{4}$ (Fig. $1 \mathrm{~b}$ and c). To examine whether or not this absorbance decrease is associated with spirocyclization of 2-thio $\mathrm{RB}$, we synthesized 2-Me, 2-OH, and 2-SMe rhodamine B and examined their reactivity with sulfane sulfur (Fig. S1, ESI $\dagger$ ). These compounds did not show any absorbance decrease. This result suggests that direct nucleophilic attack of $\mathrm{Na}_{2} \mathrm{~S}_{4}$ on the xanthene moiety does not occur. We also measured the ESI-MS spectrum of the reaction mixture of 2-thio $\mathrm{RB}$ and $\mathrm{Na}_{2} \mathrm{~S}_{4}$, and observed a characteristic MS peak of the spirocyclized form of 2-thio RB (Fig. 1d). These results confirmed that the absorbance decrease at $560 \mathrm{~nm}$ is due to formation of a persulfide group, followed by intramolecular spirocyclization.

Next, we examined whether this compound shows the same absorbance decrease in the presence of $5 \mathrm{mM} \mathrm{GSH}$, because GSH is reported to be present at the concentration of $0.5-10 \mathrm{mM}$ inside cells. ${ }^{12}$ The absorbance decrease upon addition of $\mathrm{Na}_{2} \mathrm{~S}_{4}$ occurred even in the presence of $5 \mathrm{mM} \mathrm{GSH}$, but the absorbance gradually recovered thereafter (Fig. S2a, ESI†). This recovery was not observed in the absence of $5 \mathrm{mM}$ GSH (Fig. S2b, ESI $\dagger$ ). So, we think that GSH reduced the intramolecular disulfide bond of the spirocycle, regenerating the open form of 2-thio RB, as shown in Fig. 1a, and thereby resulting in recovery of the absorbance. Further, since 2-thio RB is weakly fluorescent, probably due to photoinduced electron transfer $(\mathrm{PeT}){ }^{13}$ we expected that it would act as a fluorescence quencher. Therefore, we considered that 2-thio RB would be available as the reversible off/on switching moiety of a fluorescent probe for sulfane sulfur.

Accordingly, we designed and synthesized a novel fluorescent probe for sulfane sulfur, SSip-1, in which 2-thio RB serves as the Förster resonance energy transfer (FRET) acceptor (fluorescence quencher) and fluorescein as the FRET donor (Fig. 2a). The fluorescence spectrum of fluorescein has a large overlap integral with the absorption spectrum of 2-thio RB, and thus we expected that energy transfer from fluorescein to 2-thio RB would occur efficiently via FRET. $^{14}$
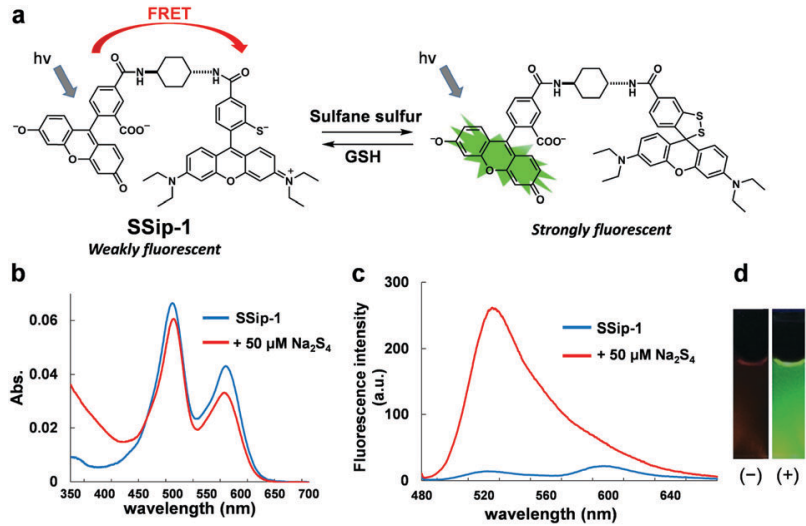

Fig. 2 (a) Chemical structure of SSip-1 and reaction mechanism with sulfane sulfur. (b and c) Changes of absorption (b) and fluorescence (c) spectra of $1 \mu \mathrm{M}$ SSip-1 before (blue) and after (red) reaction with $50 \mu \mathrm{M}$ $\mathrm{Na}_{2} \mathrm{~S}_{4}$. Both spectra were measured in $100 \mathrm{mM}$ sodium phosphate buffer (pH 7.4) containing $100 \mu \mathrm{M} \mathrm{GSH}, 0.1 \%$ DMSO as a cosolvent, and $1 \mathrm{mg} \mathrm{mL}^{-1}$ BSA. (d) Photos showing fluorescence of SSip-1 before (-) and after (+) reaction with $50 \mu \mathrm{M} \mathrm{Na}_{2} \mathrm{~S}_{4}$ (irradiated at $365 \mathrm{~nm}$ with a handy UV lamp).

The fluorescence and absorbance spectra of the probe were measured (Fig. 2b-d). The probe showed absorption maxima $\left(\lambda_{\max }\right)$ at 480 and $560 \mathrm{~nm}$, and weak fluorescence peaks derived from both fluorescein and rhodamine were also observed. Upon addition of $50 \mu \mathrm{M} \mathrm{Na}_{2} \mathrm{~S}_{4}$ to the solution, the absorbance at $560 \mathrm{~nm}$ decreased and the fluorescence at $525 \mathrm{~nm}$ increased within $1 \mathrm{~min}$. The apparent fluorescence quantum yield increased from 0.015 to 0.20 (for details, see ESI $\dagger$ ). The fluorescence intensity was well correlated to the concentration of added $\mathrm{Na}_{2} \mathrm{~S}_{4}$ in the range of $0.5-20 \mu \mathrm{M}$ in the absence of GSH (Fig. 3a). The detection limit was found to be $0.42 \mu \mathrm{M}$.

We next examined whether this probe can reversibly respond to sulfane sulfur by addition of $5 \mathrm{mM} \mathrm{GSH}$ (Fig. 3b). Treatment of SSip-1 with $50 \mu \mathrm{M} \mathrm{Na}_{2} \mathrm{~S}_{4}$ resulted in 15-fold fluorescence increase due to intramolecular spirocyclization of the FRET acceptor (2-thio RB moiety). Subsequently, the intramolecular disulfide bond of the spirocycle was slowly reduced by GSH, causing the fluorescence at $525 \mathrm{~nm}$ to decrease due to the restoration of FRET between fluorescein and 2-thio RB. The fluorescence intensity values were well correlated to the amount of added $\mathrm{Na}_{2} \mathrm{~S}_{4}$ even in the presence of $5 \mathrm{mM}$ GSH (Fig. S3, ESI $\dagger$ ). The fluorescence intensity probably depends upon the equilibrium between sulfane sulfur and GSH, and therefore this probe can monitor the concentration change of sulfane sulfur. But, to measure the exact concentration of sulfane sulfur in equilibrium with GSH, we would need to prepare a standard curve using other methods, such as LC-MS analysis. The fluorescence decrease was also correlated to the amount of added GSH (Fig. S4, ESI†). Polysulfides such as $\mathrm{Na}_{2} \mathrm{~S}_{4}$ are rapidly decomposed by GSH through thiol-disulfide exchange, releasing $\mathrm{H}_{2} \mathrm{~S} .{ }^{15}$ We found that the reversible response to sulfane sulfur could be repeated at least four times with no loss of dynamic range of the probe, i.e., the fluorescence intensity maximum (Fig. S5, ESI $\dagger$ ). As the addition of $\mathrm{Na}_{2} \mathrm{~S}_{4}$ was repeated, we interestingly found that the fluorescence intensity did not decrease completely to the 


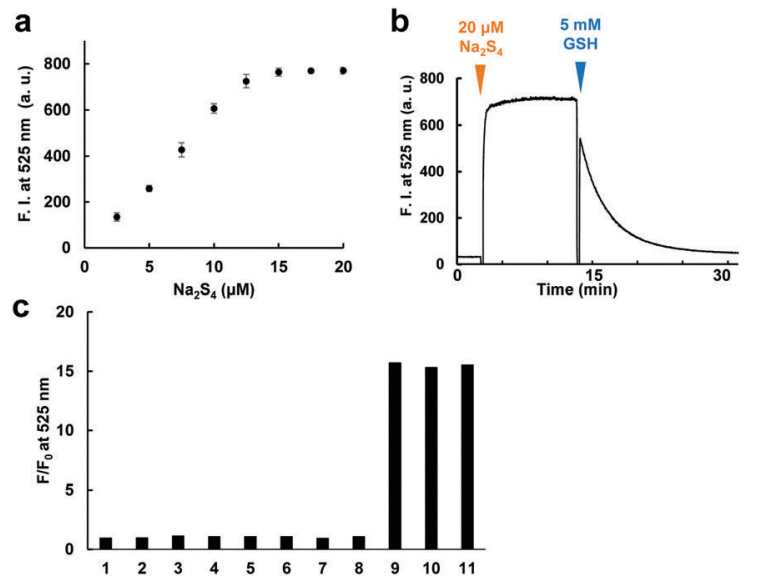

Fig. 3 (a) Fluorescence intensity of SSip-1 $(1 \mu \mathrm{M})$ upon addition of various concentrations of $\mathrm{Na}_{2} \mathrm{~S}_{4}(0,2.5,5,7.5,10,12.5,15,17.5,20 \mu \mathrm{M})$. Fluorescence intensity was measured 60 min after addition of $\mathrm{Na}_{2} \mathrm{~S}_{4}$. (b) Fluorescence intensity change of SSip-1 after addition of $20 \mu \mathrm{M} \mathrm{Na}{ }_{2} \mathrm{~S}_{4}$ followed by $5 \mathrm{mM} \mathrm{GSH}$. The fluorescence intensity $(525 \mathrm{~nm})$ of the reaction mixture was monitored. $\lambda_{\text {ex }}=470 \mathrm{~nm}$. SSip-1 was treated with $20 \mu \mathrm{M} \mathrm{Na}_{2} \mathrm{~S}_{4}$ and then reduced with $5 \mathrm{mM} \mathrm{GSH}$. (c) Fluorescence enhancement of $1 \mu \mathrm{M}$ SSip-1 measured in $100 \mathrm{mM}$ sodium phosphate buffer $(\mathrm{pH} 7.4)$ with $10 \mu \mathrm{M}$ TCEP (to reduce the intermolecular disulfide bond of dimerized SSip-1) upon addition of various thiol species. Bars represent the relative fluorescence intensities $1 \mathrm{~min}$ after addition of various thiol species. The reactions of $1 \mu \mathrm{M}$ SSip-1 with (1) $5 \mathrm{mM}$ GSH, (2) $1 \mathrm{mM} \mathrm{L-Cys,} \mathrm{(3)} 1 \mathrm{mM}$ L-Hcys, (4) $1 \mathrm{mM}$ GSSG, (5) $100 \mu \mathrm{M} \mathrm{Na}_{2} \mathrm{~S}_{2} \mathrm{O}_{3}$, (6) $100 \mu \mathrm{M} \mathrm{Na}_{2} \mathrm{SO}_{3}$, (7) $100 \mu \mathrm{M} \mathrm{Na}_{2} \mathrm{SO}_{4}$, (8) $50 \mu \mathrm{M} \mathrm{Na}_{2} \mathrm{~S}$, (9) $50 \mu \mathrm{M} \mathrm{Na}_{2} \mathrm{~S}_{2}$, (10) $50 \mu \mathrm{M} \mathrm{Na}_{2} \mathrm{~S}_{3}$ and (11) $50 \mu \mathrm{M} \mathrm{Na}_{2} \mathrm{~S}_{4}$ were examined.

original level even at $60 \mathrm{~min}$ after addition of $\mathrm{Na}_{2} \mathrm{~S}_{4}$. We confirmed that this result was not due to decomposition of the probe by measuring the fluorescence increase upon addition of SSip-1 to premixed solutions of various concentrations of $\mathrm{Na}_{2} \mathrm{~S}_{4}$ and $5 \mathrm{mM}$ GSH (Fig. S6, ESI $\dagger$ ). We consider that sulfane sulfur may be gradually stored in the reaction mixture due to stabilization by $\mathrm{GSH}$, i.e., sulfane sulfur may exist in the form of glutathione persulfides, etc. We also examined the selectivity of SSip-1 for sulfane sulfur. As shown in Fig. 3c, the fluorescence enhancement of SSip-1 occurred only upon addition of polysulfides. No fluorescence increase was observed in the presence of other biothiols, inorganic sulfur compounds, or $\mathrm{H}_{2} \mathrm{~S}$. Thus, SSip-1 shows high selectivity for sulfane sulfur.

We next examined the suitability of SSip-1 for fluorescence imaging of intracellular sulfane sulfur. Since this probe was expected to be membrane-impermeable because of its hydrophilicity derived from the carboxyl group and the thiol group, we synthesized diacetylated and thiol-protected SSip-1 (SSip-1 DA) to improve its membrane permeability (Fig. 4a). Indeed, this compound proved to be a membrane-permeable precursor that is converted to SSip-1 by intracellular esterases and reductive conditions probably related to GSH (Fig. S7, ESI $\dagger$ ). Therefore, A549 cells were incubated with $10 \mu \mathrm{M}$ SSip-1 DA DMEM for $1 \mathrm{~h}$ and then washed with HBSS. Upon addition of $10 \mu \mathrm{M} \mathrm{Na}{ }_{2} \mathrm{~S}_{4}$, a large intracellular fluorescence enhancement was observed, while almost no fluorescence was observed in the absence of $\mathrm{Na}_{2} \mathrm{~S}_{4}$ (Fig. S8, ESI $\dagger$ ). This fluorescence increase was directly related to the concentration of added $\mathrm{Na}_{2} \mathrm{~S}_{4}$ (Fig. S9, ESI $\dagger$ ). The result of a
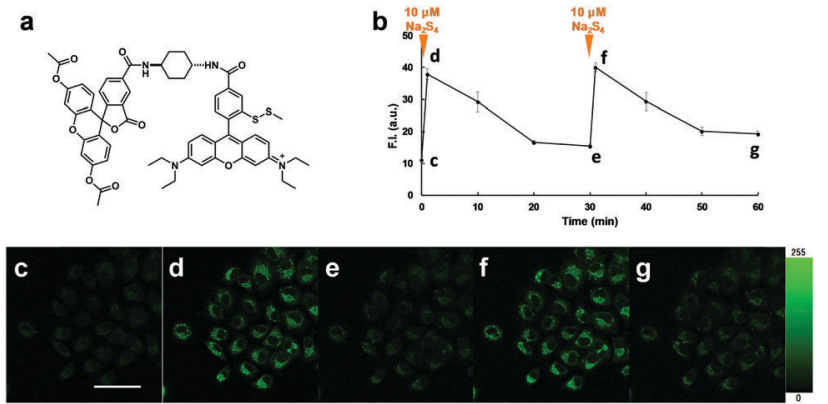

Fig. 4 (a) Chemical structure of SSip-1 DA. (b) Fluorescence intensity change of A549 cells loaded with SSip-1 DA upon repeated additions of $10 \mu \mathrm{M} \mathrm{Na}_{2} \mathrm{~S}_{4}$. Data represent the mean $\pm \mathrm{SD}(n=5)$. The mean fluorescence intensity of 5 cells is shown in the graph. $(c-g)$ Fluorescence confocal microscopic images of A549 cells loaded with SSip-1 DA upon repeated additions of $10 \mu \mathrm{M} \mathrm{Na}_{2} \mathrm{~S}_{4}$. Scale bar: $75 \mu \mathrm{m}$.

CCK-8 assay also showed that SSip-1 DA exhibited no cytotoxicity at concentrations up to $100 \mu \mathrm{M}$ (Fig. S10, ESI $\dagger$ ). Then, we investigated whether this probe can reversibly detect sulfane sulfur in living cells. A549 cells were loaded with $10 \mu \mathrm{M}$ SSip-1 DA for $1 \mathrm{~h}$; almost no fluorescence was observed (Fig. 4c). However, addition of $10 \mu \mathrm{M} \mathrm{Na}_{2} \mathrm{~S}_{4}$ to the extracellular medium resulted in a substantial fluorescence increase in the intracellular region within $1 \mathrm{~min}$ (Fig. 4d). During further incubation for $30 \mathrm{~min}$, the intracellular fluorescence decreased to the basal level (Fig. 4e). Second addition of $10 \mu \mathrm{M} \mathrm{Na}_{2} \mathrm{~S}_{4}$ to the medium resulted in a fluorescence increase in the intracellular region to the same level as that after the first addition of $10 \mu \mathrm{M} \mathrm{Na} \mathrm{S}_{4}$ (Fig. 4f). The fluorescence intensity again decreased to the original level when the cells were incubated for another $30 \mathrm{~min}$ (Fig. $4 \mathrm{~b}$ and g). These results confirm that SSip-1 can reversibly detect sulfane sulfur in living cells. The detection cycle could be repeated at least three times (data not shown).

Finally, we applied SSip-1 DA to primary-cultured hippocampal astrocytes. It is reported that polysulfides induce $\mathrm{Ca}^{2+}$ influx in rat astrocytes by activating transient receptor potential (TRP) A1 channels through modification of N-terminal cysteine residues. ${ }^{16,17}$ However, it has not been established whether the activation of TRPA1 channels occurs simultaneously with the increase in intracellular concentration of polysulfides. So, we attempted to visualize the concentration change of intracellular polysulfide in primary-cultured hippocampal astrocytes and also to monitor activation of TRPA1 channels induced by $\mathrm{Na}_{2} \mathrm{~S}_{3}$ by fluorescence imaging of intracellular $\mathrm{Ca}^{2+}$. In this experiment, we used $\mathrm{Na}_{2} \mathrm{~S}_{3}$ as a sulfane sulfur donor because $\mathrm{H}_{2} \mathrm{~S}_{3}$ is a dominant form of $\mathrm{H}_{2} \mathrm{~S}_{n}$ produced by $3 \mathrm{MST}^{6}{ }^{6} \mathrm{Na}_{2} \mathrm{~S}_{3}$ is known to possess almost the same properties as $\mathrm{Na}_{2} \mathrm{~S}_{4}$ as a sulfane sulfur donor. Hippocampi dissected from embryonic rats were dispersed, and the cells were cultured for 14 days. The cultured astrocytes were loaded with either $10 \mu \mathrm{M}$ SSip-1 DA or $2 \mu \mathrm{M}$ Fluo- 4 AM $\left(\mathrm{Ca}^{2+}\right.$ indicator) and then $\mathrm{Na}_{2} \mathrm{~S}_{3}$ was applied. Changes in sulfane sulfur and $\mathrm{Ca}^{2+}$ concentrations were monitored as changes of fluorescence intensity relative to the corresponding control image acquired before stimulation (Fig. 5). SSip-1 DA successfully visualized the increase of sulfane sulfur concentration in astrocytes, 

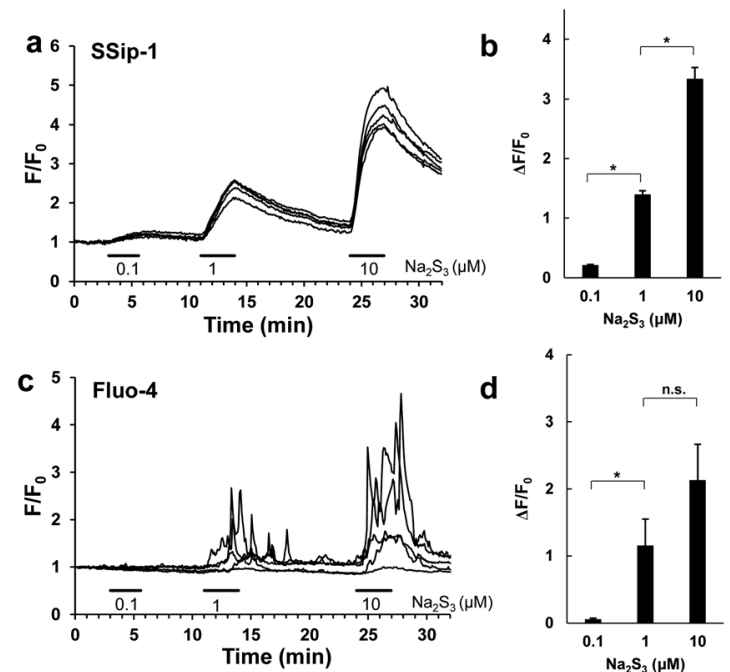

Fig. 5 Changes in intracellular polysulfide and $\mathrm{Ca}^{2+}$ levels induced by $\mathrm{Na}_{2} \mathrm{~S}_{3}$ in cultures of hippocampal astrocytes. (a) Increases in intracellular polysulfide levels induced by addition of $\mathrm{Na}_{2} \mathrm{~S}_{3}$ to the extracellular medium. $\mathrm{Na}_{2} \mathrm{~S}_{3}(0.1,1$ or $10 \mu \mathrm{M})$ was applied to astrocytes pre-loaded with $10 \mu \mathrm{M}$ SSip-1 DA for 60 min at $37^{\circ} \mathrm{C}$. (b) Graphic representation of (a). (c) Increases in intracellular $\mathrm{Ca}^{2+}$ levels induced by addition of $\mathrm{Na}_{2} \mathrm{~S}_{3}$ to the extracellular medium. $\mathrm{Na}_{2} \mathrm{~S}_{3}$ was applied to astrocytes pre-loaded with $2 \mu \mathrm{M}$ Fluo-4 AM for $30 \mathrm{~min}$ at room temperature. (d) Graphic representation of (c). All data represent the mean $\pm \operatorname{SEM}(n=10) . *: p<0.05$.

which was dependent on the amount of added $\mathrm{Na}_{2} \mathrm{~S}_{3}$ (Fig. 5a and b). Reversibility of SSip-1 was also confirmed, i.e., the fluorescence intensity increased after addition of $\mathrm{Na}_{2} \mathrm{~S}_{3}$, then decreased upon wash-out of the extracellular medium. This procedure was repeated three times with different amounts of added $\mathrm{Na}_{2} \mathrm{~S}_{3}$. The intracellular $\mathrm{Ca}^{2+}$ concentrations also changed, dependently on the amount of added $\mathrm{Na}_{2} \mathrm{~S}_{3}$ (Fig. $5 \mathrm{c}$ and d). These results indicate that the extent of $\mathrm{Ca}^{2+}$ influx was closely related to the intracellular concentration of polysulfide in the astrocytes.

In summary, we designed a reversible off/on fluorescent probe for sulfane sulfur based on the findings that 2-thio RB reacts with sulfane sulfur to afford an intramolecular spirocycle, and this spirocycle is cleaved in the presence of $5 \mathrm{mM}$ GSH (a typical intracellular concentration). The spirocyclization is accompanied with a marked decrease of absorbance at $560 \mathrm{~nm}$. Since 2-thio RB is weakly fluorescent, we considered that it could act as a quencher, and could serve a reversible fluorescence off/on switching moiety. Therefore, we synthesized SSip-1, in which 2-thio RB serves as a FRET acceptor and fluorescein as a FRET donor. We found that SSip-1 reacts with sulfane sulfur to afford a highly fluorescent, intramolecularly spirocyclized structure, which is subsequently cleaved by intracellular GSH to regenerate the weakly fluorescent open form. This probe successfully and repeatedly visualized concentration changes (both increase and decrease) of intracellular sulfane sulfur in living cells. We expect that this probe will be a useful tool for exploring the physiological functions of sulfane sulfur.

\section{Notes and references}

1 J. I. Toohey, Anal. Biochem., 2011, 413, 1.

2 T. Ida, T. Sawa, H. Ihara, Y. Tsuchiya, Y. Watanabe, Y. Kumagai, M. Suematsu, H. Motohashi, S. Fujii, T. Matsunaga, M. Yamamoto, K. Ono, N. O. Devarie-Baez, M. Xian, J. M. Fukuto and T. Akaike, Proc. Natl. Acad. Sci. U. S. A., 2014, 111, 7606.

3 P. K. Yadav, M. Martinov, V. Vitvitsky, J. Seravalli, R. Wedmann, M. R. Filipovic and R. Banerjee, J. Am. Chem. Soc., 2016, 138, 289.

4 A. K. Mustafa, M. M. Gadalla, N. Sen, S. Kim, W. Mu, S. K. Gazi, R. K. Barrow, G. Yang, R. Wang and S. H. Snyder, Sci. Signaling, 2009, 96, ra72.

5 R. Greiner, Z. Pálinkás, K. Bäsell, D. Becher, H. Antelmann, P. Nagy and T. P. Dick, Antioxid. Redox Signaling, 2013, 15, 1749.

6 Y. Kimura, Y. Toyofuku, S. Koike, N. Shibuya, N. Nagahara, D. Lefer, Y. Ogasawara and H. Kimura, Sci. Rep., 2015, 5, 14774.

7 C. M. Park, L. Weerasinghe, J. J. Day, J. M. Fukuto and M. Xian, Mol. BioSyst., 2015, 11, 1775.

8 K. Ono, T. Akaike, T. Sawa, Y. Kumagai, D. Wink, D. J. Tantillo, A. J. Hobbs, P. Nagy, M. Xian, J. Lin and J. M. Fukuto, Free Radical Biol. Med., 2014, 77, 82.

9 W. Chen, C. Liu, B. Peng, Y. Zhao, A. Pacheco and M. Xian, Chem. Sci., 2013, 4, 2892.

10 C. Liu, W. Chen, W. Shi, B. Peng, Y. Zhao, H. Ma and M. Xian, J. Am. Chem. Soc., 2014, 136, 7257.

11 W. Chen, E. W. Rosser, T. Matsunaga, A. Pacheco, T. Akaike and M. Xian, Angew. Chem., Int. Ed., 2015, 54, 13961.

12 G. Wu, Y.-Z. Lu, S. Yang, J. R. Lupton and N. D. Turner, J. Nutr., 2004, 134, 489.

13 Y. Urano, M. Kamiya, K. Kanda, T. Ueno, K. Hirose and T. Nagano, J. Am. Chem. Soc., 2005, 127, 4888.

14 Z. X. Han, X. B. Zhang, Z. Li, Y. J. Gong, X. Y. Wu, Z. Jin, C. M. He, L. X. Jian, J. Zhang, G. L. Shen and R. Q. Yu, Anal. Chem., 2010, 82, 3108.

15 D. Liang, H. Wu, M. W. Wong and D. Huang, Org. Lett., 2015, 17, 4196.

16 Y. Kimura, Y. Mikami, K. Osumi, M. Tsugane, J. Oka and H. Kimura, FASEB J., 2013, 27, 2451.

17 Y. Hatakeyama, K. Takahashi, M. Tominaga, H. Kimura and T. Ohta, Mol. Pain, 2015, 11, 24 